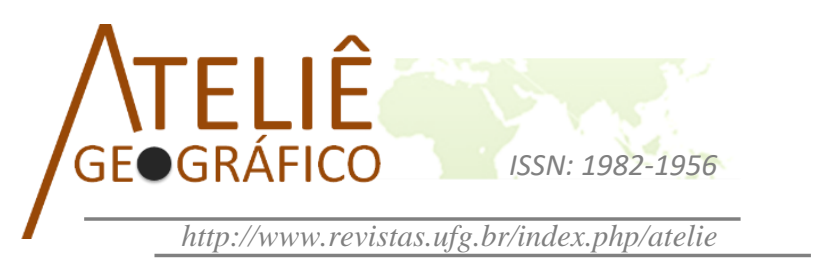

\title{
Risco socioambiental à leptospirose humana no Aglomerado Urbano Metropolitano de Curitiba, Paraná-Brasil: uma proposta metodológica apoiada em SIG
}

\author{
Social-Environmental risk to human leptospirosis in Urban \\ Agglomeration Metropolitan of Curitiba, Paraná-Brazil: a \\ methodological proposal supported by GIS
}

Risque socio-environnemental pour la leptospirose humaine
l'Agglomération Urbaine Métropolitaine de Curitiba,
Paraná - Brésil: une proposition méthodologique basée sur
le SIG

\author{
Elaiz Aparecida Mensch Buffon \\ Universidade Federal do Paraná \\ eambuffon@gmail.com
}

\author{
Francisco de Assis Mendonça \\ Universidade Federal do Paraná \\ chico@ufpr.br
}

\begin{abstract}
Resumo
$\mathrm{O}$ conhecimento das áreas de risco socioambiental à leptospirose humana pode auxiliar no processo de tomada de decisão por gestores. A quantificação e o mapeamento desse risco por meio de um índice sintético possibilitam uma análise integrada da problemática. Nesse sentido, a presente pesquisa tem como objetivo propor e avaliar uma metodologia para o mapeamento do risco socioambiental à leptospirose humana por meio de um índice sintético que considera as vulnerabilidades e a exposição à doença. A proposta foi aplicada no Aglomerado Urbano Metropolitano de Curitiba, visando apresentar uma análise no nível de desagregação intraurbano e intrametropolitano, considerando um cenário de 2007 a 2013. Foram utilizadas técnicas estatísticas associada a cartografia de síntese (análise multicritério, cálculo álgebra de mapas, cálculo de incidência e matriz de cruzamentos), que culminaram nos seguintes resultados: 1) a metodologia se mostrou eficaz para sintetizar um grande número de dados, que possibilitou reduzir fenômenos complexos de inter-relações em mensagens simples e, 2) os maiores índices de risco socioambiental estão localizados tanto em regiões periféricas,
\end{abstract}


especialmente nas áreas de conurbação, como, em regiões pericentrais, que representam bolsões de pobreza, especialmente na cidade de Curitiba.

Palavras-chave: Saúde humana; Vulnerabilidades; Exposição; Análise espacial; Cidade.

\begin{abstract}
The knowledge of the areas of risk to socio-environmental human leptospirosis can assist in the process of decision-making by managers. The quantification and mapping of this risk by means of a synthetic index enables an integrated analysis of the issue. Thus, this research aims to propose and evaluate a methodology for mapping of the risk to environmental human leptospirosis, by means of a synthetic index that considers the vulnerabilities and exposure to disease. The proposal has been applied in the Urban Agglomeration Metropolitan of Curitiba, aiming to present an analysis at the level of disaggregation intra and intrametropolitan, considering a scenario from 2007 to 2013. We used statistical techniques associated with the mapping of synthesis (multicriteria analysis, maps algebra, calculation of incidence and matrix crossroad), which culminated in the following results: 1) the methodology has proven effective for synthesizing a large number of data, which allowed to reduce complex phenomena of inter-relationships in simple messages and, 2) the greatest risk indexes socio-environmental are located both in peripheral regions, especially in the areas of conurbation, but also in regions near the center, representing pockets of poverty, especially in the city of Curitiba.
\end{abstract}

Keywords: Human leptospirosis; vulnerabilities; exposure; Geographic Information Systems; Metropolis of Curitiba.

\title{
Résumé
}

La connaissance des secteurs de du risque socio-environnemental la leptospirose humaine peut contribuer au processus de prise de décisions par les gestionnaires. La quantification et la cartographie de ce risque au moyen d'un indice synthétique permet une analyse intégrée de la question. En ce sens, cette recherche vise à proposer et d'évaluer une méthodologie de quantification et de cartographie du risque socio-environnemental la leptospirose humaine, au moyen d'un indice synthétique qui tient compte de la vulnérabilité et l'exposition à la maladie. La proposition a été appliqué l'Agglomération Urbaine Métropolitaine de Curitiba, visant à présenter une analyse au niveau de désagrégation des secteurs censitaires, considérant un scénario de 2007 à 2013. Nous avons utilisé des techniques statistiques associés à la cartographie de synthèse (analyse multicritère, algèbre de cartes, calcul de l'incidence et l'éventail des jonctions), qui a abouti à des résultats suivants: 1) la méthode s'est avérée efficace pour la synthèse d'un grand nombre de données, ce qui a permis de réduire les phénomènes complexes de relations dans des messages simples d'utilisation collective de la société et, 2) le plus grand risque sont les deux indices socio-environnemental situé dans les régions périphériques, en particulier dans les domaines de l'agglomération, mais aussi dans les régions à proximité du centre, représentant des poches de pauvreté, celui-ci en particulier dans la ville de Curitiba.

Mots-clés: Leptospirose humaine; vulnérabilités; exposition; Système d'information géographique; Métropolis de Curitiba. 


\section{Introdução}

Com a industrialização no Brasil, muitos fenômenos de ordem social, econômica, ambiental se alteraram, e assim geraram diversos impactos para a sociedade. A saúde humana dos citadinos, aspecto primordial na análise da qualidade de vida, é ameaçada por doenças das mais variadas naturezas, que podem estar diretamente ligadas com os problemas socioambientais urbanos.

As doenças de veiculação hídrica, em suas diversas categorias: fecal-oral (Hepatite A, Febre Tifoide, Diarreias), vetor (Dengue) e transmissão hídrica (leptospirose humana) (MARA \& FEACHEM, 1999), são determinantes negativos na condição de saúde humana. Para compreender a configuração e a manifestação dessas doenças, segundo Rojas (2003) é necessário relacionar os diversos fatores, sociais, econômicos, políticos, ambientais, tecnológicos e a biologia humana (MOREIRA \& LOBOS, 2011).

Os diversos modelos conceituais de saúde, por meio de indicadores de ambiente e saúde, lançados pela Organização Mundial da Saúde na década de 1990 e 2000, são exemplos de referenciais teórico-metodológicos (MONTOYA, et al., 2011). O Modelo GEO-SAÚDE, um dos últimos a ser lançado, associa os conceitos de risco e vulnerabilidade na análise dos indicadores e criação de índices (MONTOYA, et al., 2011). No entanto, cabe ressaltar que os conceitos de vulnerabilidade e risco são diversos e se sustentam em distintas orientações epistemológicas (CUTTER, 1996).

Neste trabalho adota-se a perspectiva de Mendonça (2010, p.156), entendendo que, de maneira intrínseca aos riscos identificam-se as vulnerabilidades, e dessa forma, "explicitam diferentes condições de exposição e de fragilidade de grupos sociais aos riscos". Em estudos epidemiológicos, o termo risco, têm sido utilizados como a probabilidade de acontecimentos de uma morbidade ou mortalidade, por meio da passagem do estado de saúde para o estado de doença (LAST, 1989, apud CASTIEL et al., 2010).

Importante contribuição ao conceito de risco aplicado a saúde foi apresentada pela Organização Mundial da Saúde (OMS) no ano de 1988. Conforme aponta Barbosa (1993, p.509) o termo risco foi interpretado pela OMS da seguinte forma:

É a probabilidade de ocorrência de um resultado desfavorável, de um dano ou de um fenômeno indesejado. Dessa forma, estima-se o risco ou a probabilidade de que uma doença exista, através dos coeficientes de incidência e prevalência. Considera-se "fator de risco" de um dano de toda característica ou circunstância que acompanha um aumento da probabilidade de ocorrência de um fato indesejado, sem que o dito fator tenha que intervir necessariamente em sua causalidade

Com base nesse aporte conceitual, utilizou-se nesta pesquisa uma abordagem integradora dos riscos e vulnerabilidades, a partir de um viés espacial. Sendo assim, essa 
pesquisa é uma análise centrada na manifestação de uma doença (leptospirose humana), aqui compreendida como uma consumação dos riscos sociais e naturais.

Desse modo, o recorte espacial adotado nesta pesquisa é o Aglomerado Urbano Metropolitano de Curitiba, que se caracteriza por apresentar uma mancha urbana contínua (Figura 1). Embasou-se na proposta de Firkowski (2002) para definir o conjunto de cidades que compõem o aglomerado urbano: Almirante Tamandaré, Araucária, Campina Grande do Sul, Campo Largo, Campo Magro, Colombo, Curitiba, Fazenda Rio Grande, Pinhais, Piraquara, Quatro Barras e, São José dos Pinhais.

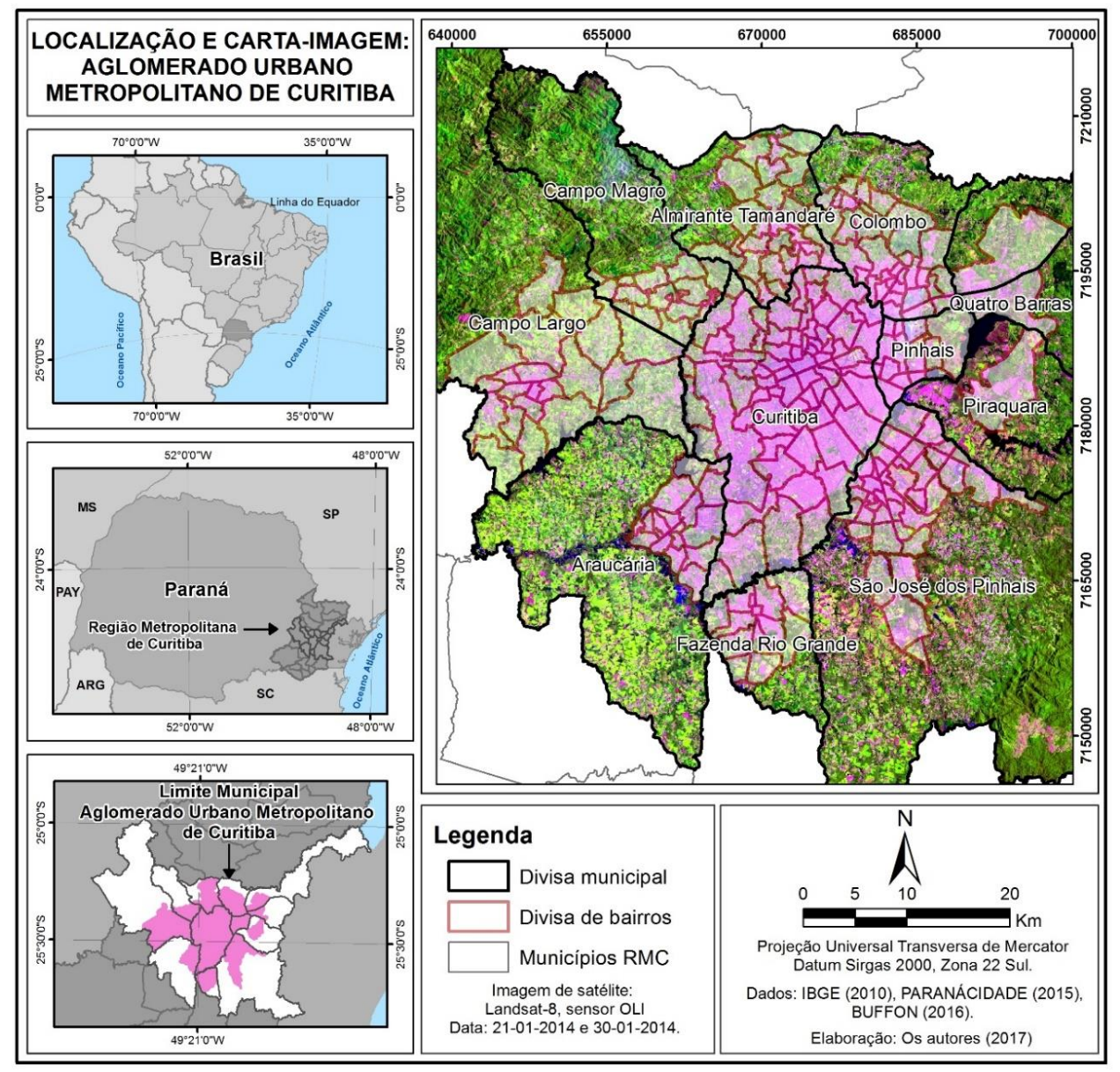

Figura 1: Aglomerado Urbano Metropolitano de Curitiba - localização e identificação da mancha urbana.

Elaboração: BUFFON, E. A. M; MENDONÇA, F. A. (2017). 
Sob essa perspectiva, objetivou-se com a presente pesquisa analisar o risco socioambiental à leptospirose humana no Aglomerado Urbano Metropolitano de Curitiba, na escala espacial do intraurbano e intrametropolitano. Para isso, propôs-se uma metodologia para o mapeamento do risco socioambiental à leptospirose humana, visando auxiliar em políticas públicas de controle e combate à doença por meio desse índice sintético.

\section{Metodologia}

O desenvolvimento desta pesquisa, que relaciona a problemática socioambiental urbana a partir da dimensão de riscos e vulnerabilidades, utiliza da abordagem do Sistema Ambiental Urbano (MENDONÇA, 2004). Apoia-se na abordagem sistêmica na perspectiva hipotético-dedutiva, sob a ótica da análise espacial, com o uso de um vasto conjunto de métodos e técnicas pautados na estatística e na cartografia.

\section{A construção da base de dados}

Os dados utilizados como indicadores de vulnerabilidades foram embasados na revisão de literatura realizada por Buffon (2016), e englobam características sociais, ambientais, demográficas e econômicas. Com exceção dos dados da variável - áreas de inundação - todas os demais foram levantados no banco de dados do Instituto Brasileiro de Geografia e Estatística (IBGE), disponibilizados por meio dos resultados do Censo de 2010, no nível de desagregação de setores censitários. É importante mencionar que para as cidades de Campo Largo, Campo Magro, Almirante Tamandaré. Campina Grande do Sul, Quatro Barras e Piraquara, o total de população por bairros não estava disponível. Os dados da variável - áreas de inundação - foram disponibilizados em formato vetorial (shapefile) pelo Instituto de Águas do Paraná.

Os dados de casos confirmados de leptospirose humana foram solicitados para a Secretaria da Saúde - Paraná (SESA) e para cada uma das Secretarias Municipais. Quanto ao recorte temporal, baseou-se na disponibilidade dos dados, considerando que no ano de 2007 se iniciou um novo sistema de notificação de Leptospirose no Estado do Paraná. Sendo assim, definiu-se o seguinte recorte temporal: 2007 a 2013.

Os dados primários, utilizados para checagens dos mapeamentos, foram obtidos por meio de trabalhos de campo realizados nos dias 12/08/2015, 14/08/2015 e $22 / 08 / 2015$. Os dados foram obtidos por fotografias realizadas no local, de modo a ilustrar e documentar as paisagens.

As bases cartográficas utilizadas nesta pesquisa para representar os limites municipais e de setores censitários foram coletadas junto ao banco de dados do IBGE, correspondentes a base do Censo de 2010. A base de limites de bairros utilizada foi disponibilizada por Buffon (2016), que integrou bases cartográficas de diversas fontes, a 
fim de obter uma única camada de informação. Além disso, aplicou os procedimentos de consistência topológica para garantir a melhor qualidade dos dados geoespaciais.

Para a elaboração dos mapeamentos foram utilizadas técnicas estatísticas e de Sistema de Informações Geográficas (SIG's), com auxílio do software ArcGIS 10.3, da empresa ESRI. Essas técnicas e métodos serão descritas a seguir para cada um dos mapeamentos.

\section{Mapeamento da vulnerabilidade social}

O mapeamento da vulnerabilidade social à leptospirose humana foi realizado com base na técnica de cartografia de síntese (MARTINELLI 1991, 2003; SAMPAIO, 2012; BUFFON, 2016; BUFFON, 2017), por meio da análise multicritério e álgebra de mapas (dados vetoriais). Os passos aplicados nos mapeamentos foram: 1) a normalização dos dados, 2) a padronização dos valores, 3) a eleição e ponderação das variáveis, e 4) o processo de síntese dos valores (SAMPAIO, 2012).

De acordo com Sampaio (2012) "o processo de normalização é definido como a relação entre duas variáveis", no caso desta pesquisa o total de domicílios por setor censitário e a variável em análise. A equação 1 expressa essa relação:

$$
V n=\frac{V 1}{V 2}(\text { Equação 1) }
$$

onde: Vn corresponde a variável normalizada, V1 a variável a ser normalizada e V2 a variável normalizadora.

Já a variável normalizada padronizada, conforme ressalta Sampaio (2012) pode ser obtida pela aplicação da equação 2 , a saber:

$$
V n p=\frac{V n-V m i n}{V \max -V \min }(\text { Equação 2) }
$$

onde Vnp é a variável normalizada padronizada, Vn a variável a ser padronizada (podendo ser normalizada ou não), VMin o menor valor da série de dados e VMax o maior valor da série de dados, sendo portanto VMax - VMin igual a amplitude (h).

Por fim, Sampaio (2012) frisa que esse processo de síntese em concepção geral é expressado pela equação 3:

$$
\text { Sintese }=\sum_{i=1}^{n} V_{n p p i}
$$

(Equação 3) 
Onde:Vnppi - representa cada uma das variáveis normalizadas padronizadas e ponderadas empregadas no processo de síntese e i varia de 1 (primeira Vnpp) até n (última Vnpp).

As variáveis utilizadas no mapeamento (Quadro 1) foram embasadas na proposta de Buffon (2017) que apresentou uma análise dos condicionantes socioambientais e indicadores de leptospirose humana.

Quadro 1: Atribuição de pesos para as categorias e sub-categorias no mapeamento de vulnerabilidade social à leptospirose humana.

\begin{tabular}{|c|c|c|c|c|c|}
\hline Ano/Dados & Categoria & Peso & Subcategoria & Peso & Variável \\
\hline \multirow{7}{*}{2010} & \multirow{5}{*}{$\begin{array}{l}\text { Características } \\
\text { dos domicílios }\end{array}$} & \multirow{5}{*}{$65 \%$} & $\begin{array}{l}\text { Rede de esgoto ou } \\
\text { fossa séptica }\end{array}$ & $25 \%$ & $\begin{array}{c}\text { Domicílios particulares } \\
\text { permanentes sem rede } \\
\text { de esgoto ou fossa } \\
\text { séptica. }\end{array}$ \\
\hline & & & Coleta de lixo & $10 \%$ & $\begin{array}{c}\text { Domicílios particulares } \\
\text { permanentes sem coleta } \\
\text { de lixo, com depósito } \\
\text { na propriedade, terreno } \\
\text { baldio, logradouro, rio, } \\
\text { lago ou mar. }\end{array}$ \\
\hline & & & Lixo em logradouro & $10 \%$ & $\begin{array}{c}\text { Domicílios particulares } \\
\text { permanentes com lixo } \\
\text { acumulado nos } \\
\text { logradouros. }\end{array}$ \\
\hline & & & Moradia/irregular & $10 \%$ & $\begin{array}{l}\text { Domicílios particulares } \\
\text { permanentes em outra } \\
\text { condição de ocupação } \\
\text { (não são próprios, } \\
\text { alugados, nem } \\
\text { cedidos). }\end{array}$ \\
\hline & & & $\begin{array}{l}\text { Abastecimento de } \\
\text { água }\end{array}$ & $10 \%$ & $\begin{array}{l}\text { Domicílios particulares } \\
\text { permanentes com fonte } \\
\text { de água proveniente de } \\
\text { poço, rios, açudes ou } \\
\text { água da chuva. }\end{array}$ \\
\hline & \multirow{2}{*}{$\begin{array}{l}\text { Características } \\
\text { dos moradores }\end{array}$} & \multirow[t]{2}{*}{$35 \%$} & Renda & $25 \%$ & $\begin{array}{l}\text { Total responsável com } \\
\text { renda até } 2 \text { salários } \\
\text { mínimos. }\end{array}$ \\
\hline & & & Alfabetização & $10 \%$ & $\begin{array}{l}\text { Total de responsáveis } \\
\text { não alfabetizados. }\end{array}$ \\
\hline
\end{tabular}

Elaboração: BUFFON, E. A. M (2017).

O índice de vulnerabilidade social a leptospirose humana proposta por Buffon (2017) é classificado em 6 classes $(k)$ : muito baixa $(0,0019-0,0750)$, baixa $(0,0751-$ $0,1336)$, média a baixa $(0,1337-0,1897)$, média a alta $(0,1898-0,2605)$, alta $(0,2506-$ $0,3650)$ e muito alta $(0,3651-0,6109)$. Os intervalos das classes para todos os mapeamentos foram realizados a partir da técnica Natural Breaks presente no software ArcGIS 10.3. 


\section{Mapeamento da incidência de leptospirose humana}

De acordo com Barbosa (1993) o uso do coeficiente de incidência permite avaliar a intensidade de ocorrência de uma morbidade sobre a população. Para calcular a taxa de incidência das doenças utilizou-se da seguinte equação (Equação 4) (BARBOSA, 1993):

$$
C . I=\frac{\text { Nůmero de casos novos } x \text { 10n }}{\text { Populaçâิo }}
$$

(Equação 4)

onde $\mathrm{n}$ equivale ao tamanho da amostra da população, e nesta pesquisa, optouse por utilizar o valor de $\mathrm{n}$ como sendo 4 , de modo que o coeficiente de incidência foi expresso por 100.000 habitantes.

O índice da incidência de leptospirose humana proposto por Buffon (2016) é classificado em seis (6) classes $(\mathrm{k})$ : sem ocorrência da doença $(0,0000)$, muito baixa $(0,0001-15,1600)$, baixa $(15,1601-40,5000)$, média a baixa $(40,5001-79,0600)$, média a alta $(79,0601-160,0000)$, alta $(160,0001-248,1000)$ e muito alta $(248,1001-$ $444,3000)$. Os intervalos das classes para todos os mapeamentos foram realizados a partir da técnica Natural Breaks presente no software ArcGIS 10.3.

\section{Mapeamento das áreas de inundações}

Conforme descrito em Suderhsa (2007) as áreas de inundações foram identificadas e delimitadas por meio de métodos de modelagem matemática, com o auxílio do modelo de simulação hidrodinâmico Mike 11 e com base em dados de vazão e de fatores associados à susceptibilidade às inundações. As áreas de inundação correspondem a cenários do ano de 2000 (dados do aerolevantamento de 1998) e tendenciais, determinando como tempo de recorrência das inundações, o período de 25 anos.

\section{Mapeamento do risco socioambiental à leptospirose humana}

A identificação das áreas de risco socioambiental a leptospirose humana foi definida considerando os mapeamentos de vulnerabilidade social e incidência da doença. $\mathrm{O}$ primeiro representa as condições de fragilidades do ambiente e da sociedade e o segundo como as áreas mais propícias a ocorrência da doença. Para obter o índice do risco socioambiental utilizou-se a matriz de cruzamento que é apresentada no quadro 2.

O primeiro cruzamento realizado foi entre os índices de vulnerabilidade social (desagregação de setores censitários) e incidência da doença (desagregação de bairros). Esse cruzamento foi realizado por meio de dados vetoriais desagregados em setores censitários. O segundo cruzamento foi realizado com o resultado do primeiro cruzamento em conjunto com as áreas de inundação. Adotou-se que, os setores que 
apresentam áreas com inundações são positivos para a ocorrência de leptospirose humana, portanto acentuam o índice de risco, e quando não apresentam áreas com inundações são negativos, logo apresentam um decréscimo no índice de risco. $\mathrm{O}$ índice do risco socioambiental à leptospirose humana foi apresentado no nível de desagregação de setores censitários, a partir de 6 classes: muito baixo, baixo, médio a baixo, médio a alto, alto e muito alto.

Quadro 2: Matriz de cruzamentos para obtenção do índice sintético de risco socioambiental a leptospirose humana.

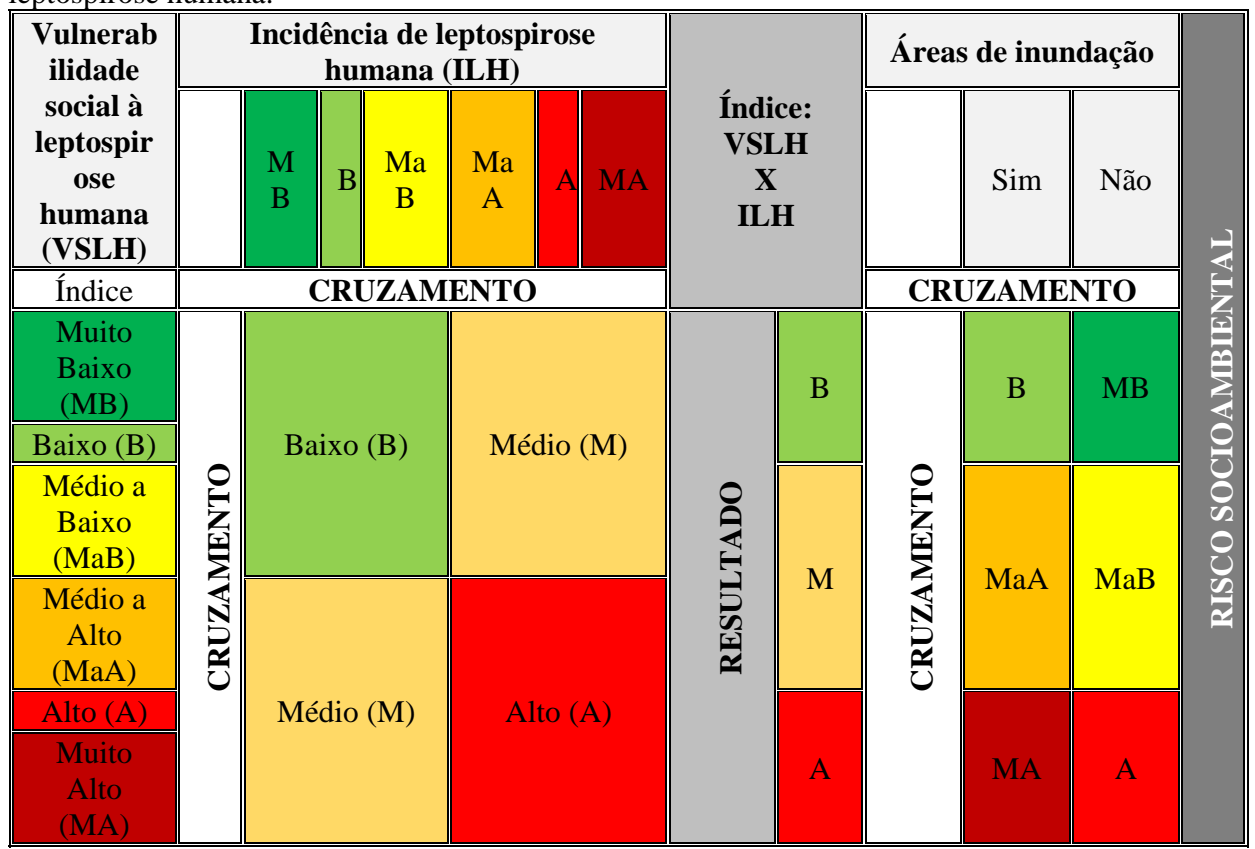

Elaboração: BUFFON, E. A. M; MENDONÇA, F. A. (2017).

Nas cidades em que não foi possível calcular o índice de incidência da doença, não se procedeu com os cruzamentos (por não apresentarem o total de população por bairro e/ou limites de bairros). Além disso, os bairros que foram caracterizados como sem ocorrência da doença, permaneceram com tal classificação, tendo em vista que não foi possível identificar a probabilidade de ocorrência da doença ao longo do tempo, e entende-se que não existe risco zero ou nulo.

\section{Resultados e discussões}

Os mapeamentos temáticos apresentados na Figura 2 demonstram a concentração dos maiores índices nas periferias geográficas das cidades e do aglomerado 
urbano. O maior índice de incidência da doença foi identificado em Curitiba, na região pericentral e na periferia. As áreas de inundações estão localizadas principalmente nas porções de periferias, especialmente associadas ao Rio Iguaçu e seus afluentes. Os maiores índices de risco socioambiental (alto e muito alto) foram identificados em 4 cidades (Curitiba, Fazenda Rio Grande, São José dos Pinhais e Colombo).

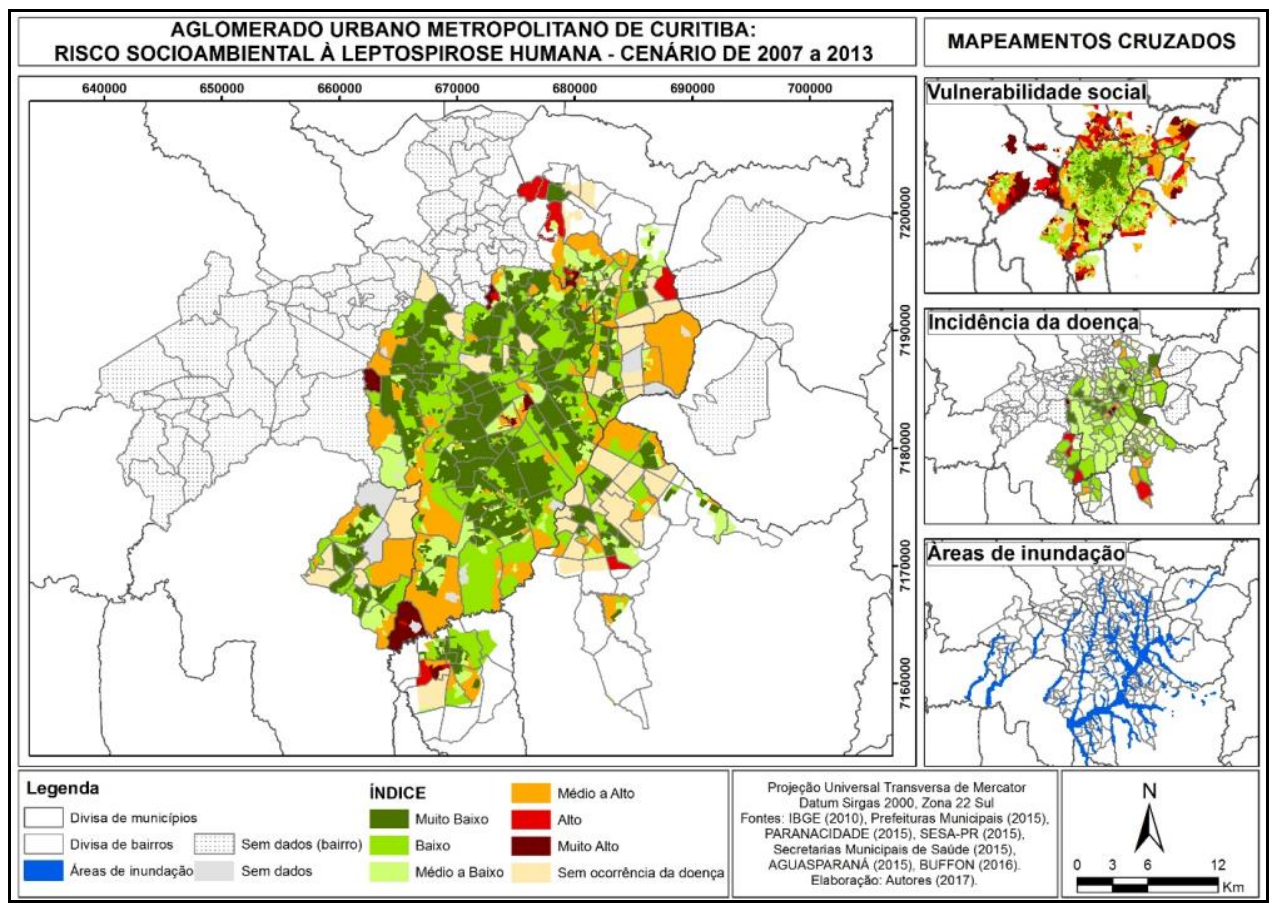

Figura 2: Leptospirose humana no Aglomerado Urbano Metropolitano de Curitiba: mapeamentos da vulnerabilidade social, incidência da doença, áreas de inundações e do risco socioambiental. Elaboração: BUFFON, E. A. M; MENDONÇA, F. A. (2017).

A análise estatística dos dados utilizados no mapeamento de vulnerabilidade social permitiu apresentar as seguintes considerações em relação a área total do aglomerado: $2,44 \%$ da área foi classificada com índice muito baixo; 6,39 no índice baixo; $17,98 \%$ com índice médio a baixo; $35,25 \%$ correspondeu ao índice médio a alto; $17,95 \%$ com índice alto e; $19,98 \%$ no índice muito alto.

Em termos de representatividade (maior porcentagem do índice) por cidade, destacam-se: no índice muito baixo (Pinhais), no índice baixo (Quatro Barras), no índice médio a baixo (Fazenda Rio Grande), no índice médio a alto (Pinhais), no índice alto (Almirante Tamandaré) e no índice muito alto (Campo Magro) (vide figura 2). As cidades de Campina Grande do Sul, Campo Largo, Campo Magro, Colombo, Fazenda Rio Grande e Piraquara, não apresentaram área com índice muito baixo (vide figura 2). 
Dentre essas, Campo Magro se destacou por, também, não apresentar condições de baixa vulnerabilidade. Na maior parte da área dessas cidades predominam os índices de médio a alto, alto e muito alto (vide figura 2).

Ainda na figura 2, observa-se que as cidades que apresentaram os maiores índices de incidência são: Curitiba (muito alto e alto) e São José dos Pinhais (alto) e Araucária (alto). No que se refere as áreas de inundações, o mapeamento permite afirmar que todas as cidades apresentam áreas com inundações e a maior concentração é nas áreas de conurbação de Curitiba com as demais cidades do aglomerado.

O mapeamento de risco socioambiental demonstrou ser mais refinado que os demais mapeamentos, permitindo assim identificar áreas prioritárias para aplicação de políticas públicas, que visem o combate, o controle e a possível erradicação da doença. Desse modo elaborou-se o quadro 3 para destacar os bairros que possuem áreas críticas no que concerne ao risco socioambiental à leptospirose humana dentro do contexto intraurbano de cada cidade do aglomerado.

Quadro 3: Cidades e seus respectivos bairros com os maiores índices de risco socioambiental no contexto intraurbano.

\begin{tabular}{|c|c|c|}
\hline CIDADE & BAIRROS & $\begin{array}{l}\text { ÍNDICE DE } \\
\text { RISCO }\end{array}$ \\
\hline Araucária & $\begin{array}{c}\text { Passaúna, Campina da Barra, Capela Velha e } \\
\text { Tindiquera }\end{array}$ & Médio a Alto \\
\hline \multirow{2}{*}{ Curitiba } & $\begin{array}{c}\text { Parolin, Prado Velho, Taboão, Riveira Guaíra e } \\
\text { Caximba }\end{array}$ & Muito Alto \\
\hline & Jardim Botânico e Pinheirinho & Alto \\
\hline \multirow{2}{*}{ Colombo } & Osasco & Muito Alto \\
\hline & Gabirobal, Boicininga, Arruda e Colônia Faria & Alto \\
\hline $\begin{array}{l}\text { Fazenda Rio } \\
\text { Grande }\end{array}$ & Santa Terezinha & Muito Alto \\
\hline Pinhais & $\begin{array}{c}\text { Emiliano Perneta, Atuba, Jardim Cláudia e } \\
\text { Weissópolis }\end{array}$ & Médio a Alto \\
\hline $\begin{array}{l}\text { São José dos } \\
\text { Pinhais }\end{array}$ & Barro Preto & Alto \\
\hline
\end{tabular}

Elaboração: BUFFON, E. A. M; MENDONÇA, F. A. (2016).

Os bairros com os maiores índices de risco não se restringiram a periferia geográfica do aglomerado urbano metropolitano, ou das suas respectivas cidades. Os bolsões de pobreza localizados nas porções centrais e pericentrais de Curitiba, Colombo 
e Fazenda Rio Grande necessitam de uma ação de controle e prevenção urgente. No contexto das porções periféricas, destacam-se as áreas de conurbação por apresentar os maiores índices de risco. Nesse sentido, Deschamps (2004, p.144) menciona que a "migração obedece a um padrão seletivo, em que as camadas mais pobres da população migrante se dirigem para as áreas periféricas da RMC, desprovidas de infra-estrutura adequada".

Com o objetivo de demonstrar de modo mais detalhado o mapeamento de risco socioambiental, realizou-se recortes espaciais que permitem observar a heterogeneidade na configuração do risco (Figura 3).

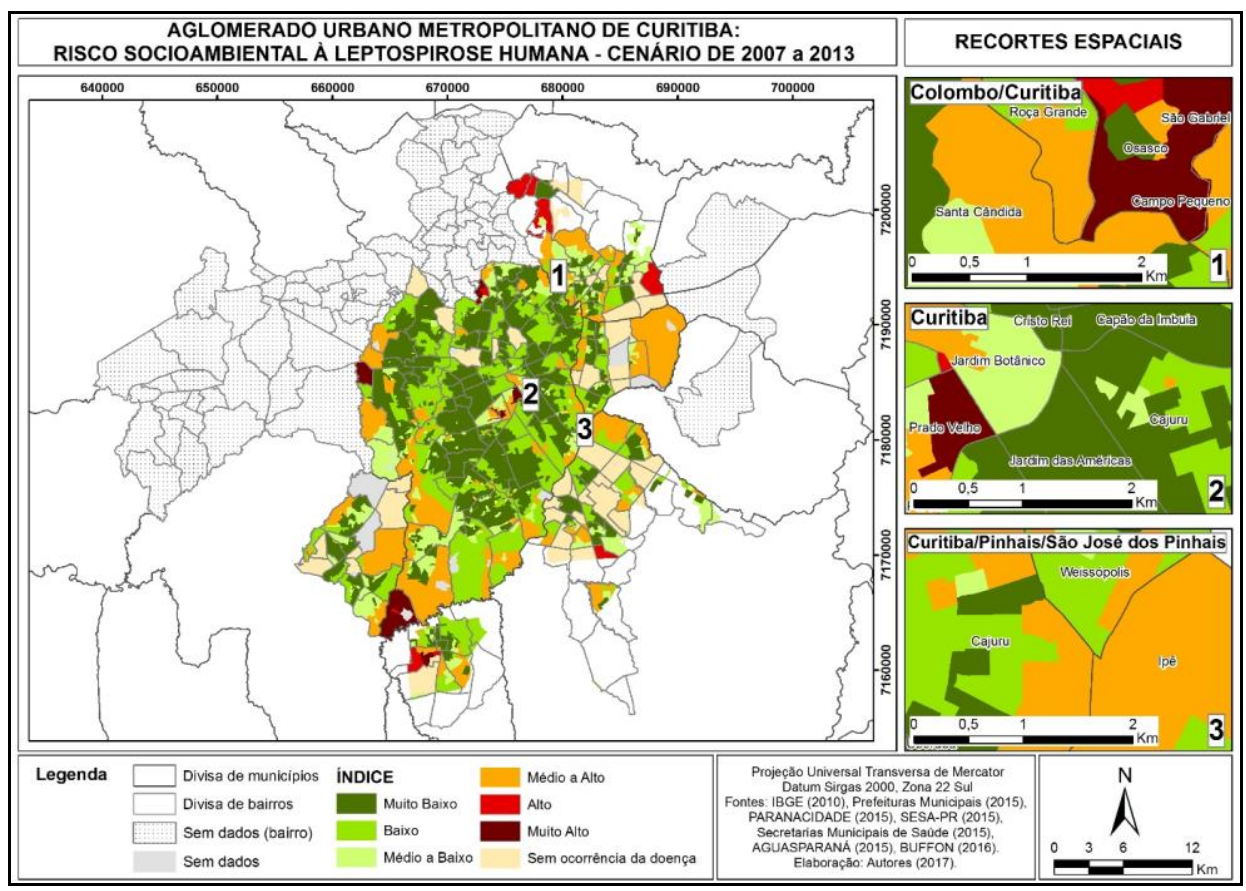

Figura 3: Leptospirose humana no Aglomerado Urbano Metropolitano de Curitiba: recortes espaciais de áreas com distintos índices de risco socioambiental.

Elaboração: BUFFON, E. A. M; MENDONÇA, F. A. (2017).

Esses recortes demonstram tanto pela heterogeneidade, bem como por suas localizações no aglomerado, que é necessário superar, conforme aponta Firkowski (2001, p. 258)

a noção de periferia limitada à sua dimensão territorial, ou seja, quando as áreas mais distantes do centro são tomadas como sinônimo de pobreza, bem como da ausência de toda a sorte de infraestrutura. Na Curitiba metropolitana, mesclam-se espaços que 
expressam a pobreza e a riqueza, o moderno e o arcaico; coexistem loteamentos de luxo com ocupações irregulares; indústrias modernas e tradicionais; o subemprego e os serviços especializados (FIRKOWSKI, 2001, p. 258).

Os recortes espaciais apresentados na figura 3 também foram utilizados na elaboração da figura 4, que visa demonstrar as paisagens observadas em distintos índices de risco. Essas paisagens são alguns exemplos de checagens do mapeamento. A foto 1, apresentada na figura 4, está inserida em contexto de risco muito alto, localizado na periferia de Colombo que é conurbada com Curitiba. Essa foto demonstra construções localizadas próximas as margens do rio e com alta vulnerabilidade social.

A foto 2 da figura 4 demonstra um exemplo do risco baixo, localizado no bairro Cajuru em Curitiba. As discrepâncias entre a paisagem observada na foto 1 e 2 são evidentes, principalmente no que concerne a infraestrutura urbana e a construção das edificações. A foto 3 da figura 5 caracteriza uma área de índice médio a alto de risco, que demonstra ser uma ocupação recente, ainda sem infraestrutura, e que constantemente é afetada por inundação, conforme relatos obtidos em campo. Esse recorte demonstra a necessidade constante de atualização dos dados do mapeamento, tendo em vista a dinamicidade do ambiente urbano.

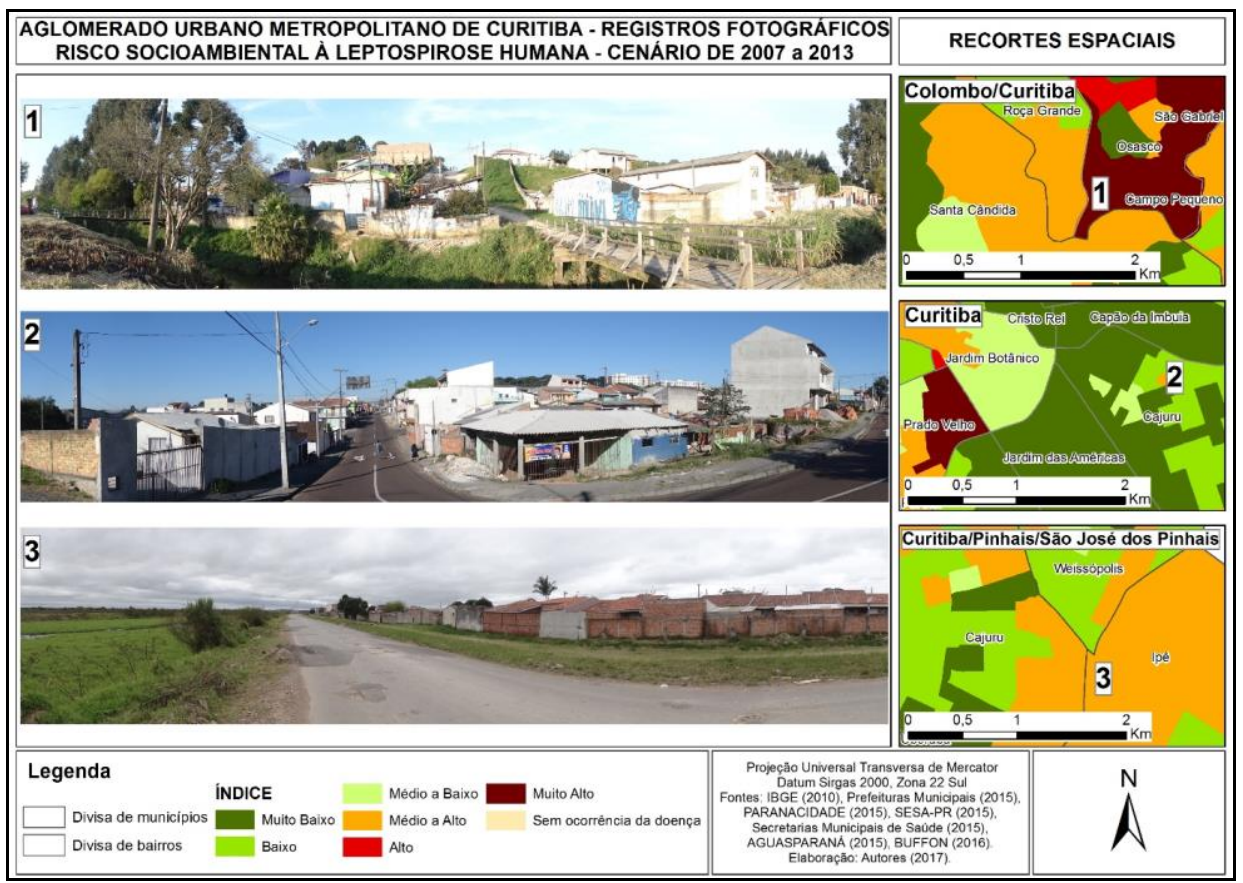

Figura 4: Leptospirose humana no Aglomerado Urbano Metropolitano de Curitiba: registros fotográficos das áreas de recortes espaciais com distintos índices de risco socioambiental. Elaboração: BUFFON, E. A. M; MENDONÇA, F. A. (2017). 
Os mapeamentos demonstraram que a cidade é caracterizada pela heterogeneidade espacial, que não obedece mais somente o padrão de centro-periferia no quesito infraestrutura e condições de vida. Foi possível observar que em alguns setores da cidade o processo de urbanização pautou-se em estudos de planejamento, em outros, o processo é amplamente rudimentar e gerado por pressões sociais e econômicas. Como exemplo, cita-se a ocupação das Áreas de Preservação Permanentes (APP's), que acometem para além da destruição da natureza, as condições de vida das populações.

Os resultados apresentados permitiram compreender que a criação de um índice sintético - risco socioambiental - possibilitou sintetizar uma grande quantidade de dados apresentados nesta pesquisa, de modo a reduzir os fenômenos complexos de interrelações em mensagens simples para uso coletivo da sociedade.

Ferraz (2002) e Paula (2003) ao abordarem a problemática da leptospirose humana em Curitiba, apontaram a necessidade de estudos na escala intraurbano como uma ferramenta para a vigilância epidemiológica, de modo a auxiliar nas ações locais. Com esse trabalho mostrou-se que a análise na escala do intraurbano associada ao uso de um índice sintético são metodologias eficazes para identificar áreas prioritárias de atenção frente ao ambiente e a saúde humana e, assim, auxiliar na tomada decisão.

\section{Conclusões}

A análise do risco socioambiental à leptospirose humana se constitui em uma abordagem multidisciplinar do ambiente e da saúde humana. A metodologia empregada (técnicas estatísticas, cartografia de síntese, cálculo da incidência e matriz de cruzamento), se mostrou eficiente e que pode ser aplicada para outras localidades, sem exigência de um grande investimento financeiro. $\mathrm{O}$ índice sintético enfatiza uma análise integrada que permite atuar frente aos diversos problemas socioambientais desencadeadores ou intensificadores da leptospirose humana, ação que se tornaria mais difícil em uma análise com índices isolados.

A configuração espacial do risco socioambiental a leptospirose humana é mais elevada (risco médio a alto, alto e muito alto) nas porções de periferias geográficas localizadas em áreas de conurbação, mas também, em bolsões de pobrezas, localizados tanto nas periferias como em regiões pericentrais. As observações empíricas realçadas neste trabalho explicam a degradação tanto da natureza como da sociedade, de modo concomitante. Enfatiza-se, por meio da análise integrada entre o ambiente e a saúde humana, que a medida que as cidades evidenciam a degradação ambiental, a sociedade demonstra se tornar mais vulnerável aos riscos.

Conclui-se que a metodologia adotada na avaliação do risco socioambiental a leptospirose humana pode ser utilizada para futuros estudos que visem auxiliar e avaliar tomadas de decisões frente a essa problemática em outras cidades. Além disso, pode ser utilizada para analisar outras doenças e problemas socioambientais, com a necessidade de inserção e alterações nos indicadores e pesos, assim como validações em campo. 
A principal expectativa com os resultados desse trabalho é que os atores políticos atuem em seus territórios de forma integrada, propondo programas que permitam atuar frente a problemática socioambiental, bem como, monitorar as ações e seus resultados. Para a área de estudo adotada neste trabalho, elencam-se as seguintes dificuldades e obstáculos que precisam ser vencidos para o melhor detalhamento dos riscos socioambientais:

1) Levantamento de dados e planos diretores das cidades de modo integrado, permitindo assim o acesso à diversas informações mais atualizadas, tais como: a delimitação de bairros, dados das variáveis sociais, econômicas, demográficas e ambientais.

2) Registros pontuais ou por unidade (bairros) da ocorrência de inundações, possibilitando assim uma análise mais refinada das áreas de inundação.

3) Disponibilizar via sistema online os dados de ocorrências das doenças por bairros, não somente por município, de modo a facilitar a execução dos estudos.

\section{Referências}

ALEIXO, N. C. R. Pelas lentes da climatologia e da saúde pública: doenças hídricas e respiratórias na cidade de Ribeirão Preto/SP. 353 f. Tese (Doutorado em Geografia) Departamento de Geografia, Faculdade de Ciências e Tecnologia, Universidade Estadual Paulista, Presidente Prudente, 2012.

ALMEIDA, L. Q. de. Vulnerabilidades socioambientais de rios urbanos: bacia hidrográfica do rio Maranguapinho, região metropolitana de Fortaleza, Ceará. $278 \mathrm{f}$. Tese (Doutorado em Geografia) - Instituto de Geociências e Ciências Exatas, Universidade Estadual Paulista, Rio Claro, 2010.

BARBOSA, L. De M. M., 1993. Glossário de Epidemiologia e Saúde. In: ROUQUAYROL, M. Z. Epidemiologia e saúde. 4. ed. Rio de Janeiro: MEDSI.

BUFFON, E. A. M. A leptospirose humana no AU-RMC (Aglomerado Urbano da Região Metropolitana de Curitiba/PR) - risco e vulnerabilidade socioambiental. Dissertação (Mestrado em Geografia) - Setor de Ciências da Terra, Programa de PósGraduação Geografia, Universidade Federal do Paraná, Curitiba, 2016, 171f.

BUFFON, E. A. M. Vulnerabilidade socioambiental à leptospirose humana no aglomerado urbano metropolitano de Curitiba, Paraná - Brasil: proposta metodológica a partir da análise multicritério e álgebra de mapas. Saúde Soc. São Paulo, 2017 (no prelo).

CASTIEL, L. D.; GUILAM, M. C. R.; FERREIRA, M. S. Correndo o risco: uma introdução aos riscos em saúde. Rio de Janeiro: Editora Fiocruz, 2010. 
COSTA, E.; et al. Formas graves de leptospirose: aspectos clínicos, demográficos e ambientais. Revista da Sociedade Brasileira de Medicina Tropical, v. 34, n.3, p.261-267, 2001.

CUTTER, S. L. Vulnerability to environmental hazard. Progress in Human Geography, v. 20, n.4, 1996, p.-529-539.

DESCHAMPS, M. V. Vulnerabilidade socioambiental na região metropolitana de Curitiba/PR. 192 f. Tese (Doutorado em Meio Ambiente e Desenvolvimento) Universidade Federal do Paraná, Curitiba, 2004.

DUARTE, G. G. F. Análise espacial da endemia de leptospirose na cidade de São Paulo, uma abordagem baseada em geoprocessamento. 148f. Dissertação (Mestrado em Geografia) - Instituto de Geociências, Universidade Estadual de Campinas, 2008.

FERRAZ, M. E. Ocorrência de leptospirose humana e fatores sócio-ambientais que influenciaram na redução dos casos entre 1997 e 2001, no distrito sanitário Cajuru, Curitiba, Paraná, Brasil. 64 f. Monografia de Especialização - Setor de Ciências Biológicas, do Departamento de Patologia Básica, Universidade Federal do Paraná, Curitiba, 2002.

FIRKOWSKI, O. L. C. de F. A nova territorialidade da indústria e o aglomerado metropolitano de Curitiba. 278 f. Tese (Doutorado em Geografia Humana) - Faculdade de Filosofia, Línguas e Ciências Humanas, Universidade de São Paulo, São Paulo, 2001.

FIRKOWSKI, O. L. C. de F. A nova lógica de localização industrial no aglomerado metropolitano de Curitiba. Revista Paranaense de Desenvolvimento, Curitiba, IPARDES, n. 103, p. 79-100, 2002.

GONÇALVES D.D.; TELES P.S.; REIS C.R.; LOPES F.M.R.; FREIRA R.L.; NAVARRO I.T.; ALVES L.A.; MULLER E.E.; FREITAS J.C. Seroepidemiology and occupational and environmental variables for leptospirosis, brucellosis and toxoplasmosis in slaughterhouse workers in the Paraná state, Brazil. Revista do Instituto de Medicina Tropical de São Paulo, v. 48, p. 135-140, 2006.

LAU C. L.; SMYTHE L. D.; CRAIG S. B.; WEINSTEIN P. Climate change, flooding, urbanisation and leptospirosis: fuelling the fire? Trans R Soc Trop Med Hyg. v. 104, n.2, p. 631-638, 2010.

MARA, D. D. \& FEACHEM, R. G. A. Water and excreta-related diseases: Unitary environmental classification. Journal of Environmental Engineering, v. 125, p.334-339, 1999.

MARTINELLI, M. Curso de cartografia temática. 1. ed. São Paulo: Contexto, 1991.

MARTINELLI, M. Mapas da geografia e cartografia temática. São Paulo: Contexto, 2003. 
MENDONÇA, F. A. Impactos socioambientais urbanos. Curitiba: Editora da UFPR, 2004.

MENDONÇA, F. A. Riscos e Vulnerabilidades socioambientais urbanos a contingência climática. Mercator, v 9, n.1, p.153-163, dez/2010.

MENDONÇA, F. A. Riscos, vulnerabilidades e resiliência socioambientais urbanas: Inovações na análise geográfica. Revista da ANPEGE, v. 7, p. 99-109, 2011.

MOHANA, A. R. M.; CUMBERBATCHB, A.; ADESIYUNC, A. A.; CHADEE, D. D. Epidemiology of human leptospirosis in Trinidad and Tobago, 1996-2007: A retrospective study. Acta Tropica v.112, p. 260-265, 2009.

MONTOYA, M. P. A; GOSSELIN, P.; HACON, S.; RUIZ, A. Indicadores de saúde ambiental para a tomada de decisões. In: In: GALVÃO, L. A. C.; FINKELMAN, J.; HENAO, S. Determinantes ambientais e sociais da saúde. Washington, DC: OPAS, 2011. p.155-181.

MOREIRA, J. C. \& LOBOS, J. O papel e os desafios dos laboratórios de saúde ambiental nas Américas. In: GALVÃO, L. A. C.; FINKELMAN, J.; HENAO, S. Determinantes ambientais e sociais da saúde. Washington, DC: OPAS, 2011.

OLIVEIRA, D. S. C.; GUIMARÃES, M. J. B.; MEDEIROS, Z. Modelo produtivo para Leptospirose. Revista de Patologia Tropical. v. 38, n.1, p.17-26, jan-mar/2009.

OLIVEIRA, T. V. dos S. Fatores Socioambientais Associados a Eventos Hidrometeorológicos Extremos na Incidência de Leptospirose no Município do Rio de Janeiro - 1997 a 2009. Um estudo de caso. 86f. Tese (Doutorado em Ciências) - Escola Nacional de Saúde Pública Sergio Arouca, Rio de Janeiro, 2013.

PAPPACHAN, M.J.; SHEELA, J.; ARAVINDAN, K.P. Relation of rainfall pattern and epidemic leptospirosis in the Indian state of Kerala. J Epidemiol Community Health, v.58, p1054 - 1055, 2004.

PAULA, E. V. Leptospirose Humana: Uma Análise Climato-Geográfica de sua manifestação do Brasil, Paraná e Curitiba (1997-2001). 116 f. Trabalho de Graduação (Bacharelado em Geografia) - Setor Ciências da Terra, Universidade Federal do Paraná, Curitiba, 2003.

ROJAS, L. I. Geografía y salud. Entre Historias, Realidades y Utopias. Caderno Prudentino de Geografia, Associação dos Geógrafos Brasileros, v. 1, n. 1, Dez./2003.

SAMPAIO, T. V. M. Diretrizes e procedimentos metodológicos para a cartografia de síntese com atributos quantitativos via álgebra de mapas e análise multicritério. Boletim de Geografia, Maringá, v. 30, n. 1, p. 121-131, 2012.

SORRE, M. A adaptação ao meio climático e biossocial - geografia psicológica. In: MEGALE, J. F. (Org.). Max Sorre. São Paulo: Ática, 1984. (Coleção Grandes Cientistas Sociais, 46). 
SUDERHSA - Instituto das Águas do Paraná. Relatório de diagnóstico - Plano da bacia do Alto Iguaçu e afluentes do Alto Ribeira. Capítulo 8 - Síntese. Curitiba: Instituto das Águas do Paraná, 2007, p.67 - 94.

TREVEJO R.T. et al. Epidemic Leptospirosis Associated with Pulmonary Hemorrhage. Nicaragua, 1995. Journal de Infectious Diseases, v. 178, p. 1457-1463, 1998.

\section{Elaiz Aparecida Mensch Buffon}

Doutoranda em Geografia na Universidade Federal do Paraná - UFPR. Mestrado em Geografia pela Universidade Federal do Paraná - UFPR - 2015. Graduação em Geografia pela Universidade Federal da Fronteira Sul - Campus Chapecó-SC - 2013. Atua no laboratório de Climatologia da UFPR. Centro Politécnico - Universidade Federal do Paraná - Av. Francisco H. dos Santos, s/n Edifício João José Bigarella. Sala 210. CEP 81531-900, Curitiba - Paraná.

E-mail: eambuffon@gmail.com

\section{Francisco de Assis Mendonça}

Doutor em Geografia pela USP - Pós-doutor pela Université de Sorbonne, London School of Hygine and Tropical Medecine e Universidad do Chile. Atualmente, é Professor Titular na UFPR e coordenador do Laboratório de Climatologia e, Pesquisador CNPQ/1A. Centro Politécnico - Universidade Federal do Paraná - Av. Francisco H. dos Santos, s/n Edifício João José Bigarella. Sala 210. CEP 81531-900, Curitiba - Paraná

E-mail: chico@ufpr.br 